\title{
SISE Semantics Interpretation Concept
}

\author{
Karel Kisza ${ }^{1}$ and Jiří Hřebíček ${ }^{2}$ \\ ${ }^{1}$ Masaryk University, Faculty of Infromatics, Botanická 68a Brno, Czech Republic \\ kkisza@mail.muni.cz \\ ${ }^{2}$ Masaryk University, Faculty of Science, Research Centre for Toxic Compounds \\ in the Environment, Kotlářská 267/2 Brno, Czech Republic
}

hrebicek@iba.muni.cz

\begin{abstract}
The development of the complete and complex Single Information Space for the Environment in the Europe (SISE) covering all interactions among environmental information and knowledge using current ICT tools is practically impossible. A common methodology of building the conceptual model of the SISE, based on ontologies and associated technologies was developed at Masaryk University. The big challenge is to find the best way how to make ontology semantics available to the user using appropriate problem solving (search) interface. The paper shows the concept of a SISE semantic interpretation, related summary of used technologies. The concept combines main advantages of ontologies and expert systems - the ontology semantics and the rule based on application are used for searching information and issuing predictions.
\end{abstract}

Keywords: Semantics, SISE, ontology, expert system.

\section{Introduction}

The paper presents a semantic idea of interpretation of theoretical model of the Single Information Space for the Environment in Europe (SISE). The Hrebicek and Pilman theoretical conceptual model [4] was compared with an upper ontology concept of the SISE [2].

The developed conceptual model of the SISE enables an implementation of the vision of development for an integrated, modern, common, shared and sustained Single European Information Space infrastructure for environmental information exchange and environmental management in Europe. The upper ontology concept of SISE was not suitable for more specific tasks (e.g. for the implementation of effects of environmental information), where the detailed level of the solution is needed. In this case it was necessary to use a more detailed model, which is much more specific than the upper ontology model (European ICT Environmental Sustainability Research, 2010) [3], [4].

Currently the research and development of shared environmental information system [3] focuses on the improvement of search results by using semantic web technologies [7]. Internet users without special knowledge in environmental topics should 
be enabled to find all available information for their special requirements by placing a request as simple as possible.

There is a big amount of various kind of ontological model in the environmental research area. These models are built both for systems in various fields (domain ontology) and for systems in business companies (application ontology). Domain ontology defines a domain based set of terms and relations, which should be independent of any problem solving method. Application ontology contains terms and relations unique to particular problem solving method. This customization allows to separates out control and procedural knowledge. The use of pre-defined domain ontologies enable users to reduce the available search space. Ontological concepts relevant to a problem domain are supplied to the user allowing them to focus their query. A more advanced interface would allow the user's query to use their terms and map that to an underlying domain.

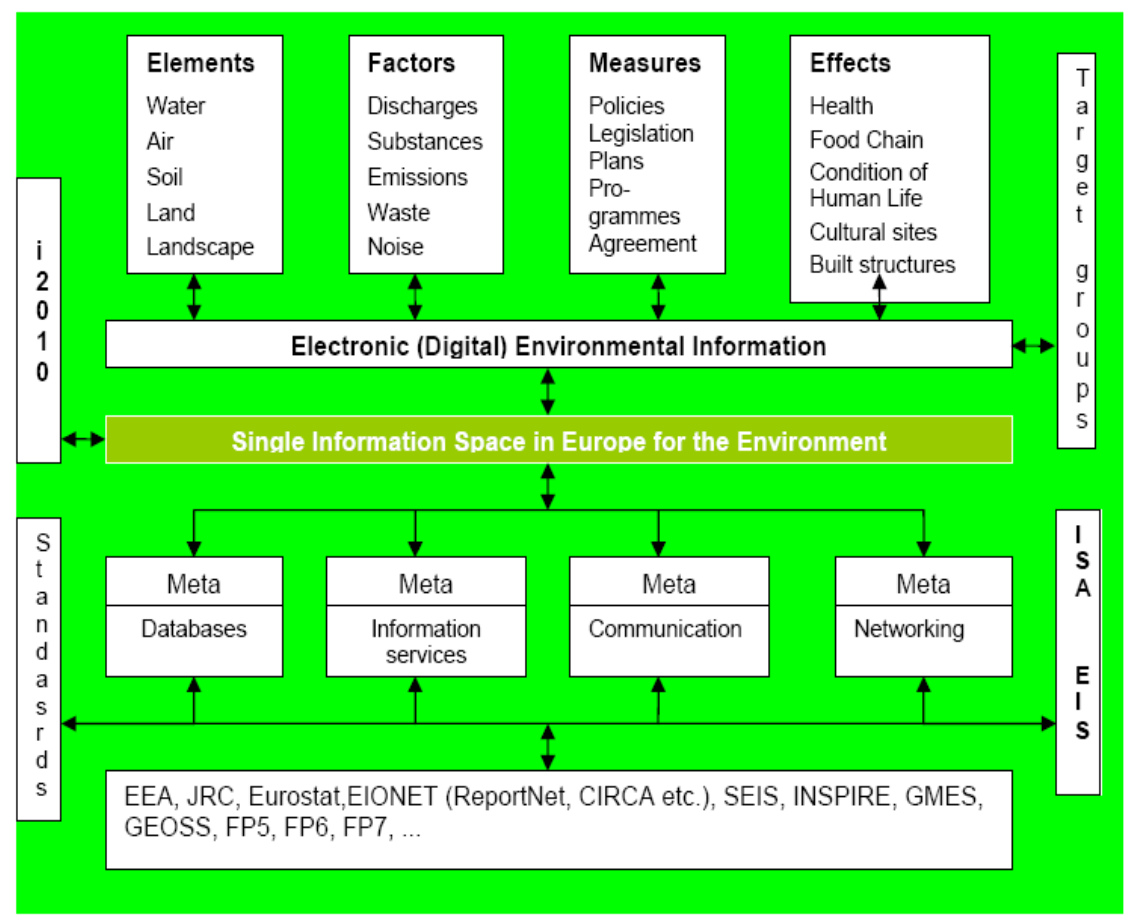

Fig. 1. Hrebicek and Pilman concept of SISE [4]

The big challenge is to find the best way how to make ontology semantics available to the user using appropriate problem solving (search) interface. Problem solving methods are defined as reasoning strategies for solving certain problem types. Here expert systems come to be used.

Since their appearance in the 1970's, rule based expert systems have found applications in a variety of domains ranging from control systems for power plants to help systems. They are mainly used for tasks like diagnostics or predictions. The typical 
scenario consists of interviewing the user in order to acquire facts that are used afterwards to generate the predictions or diagnostics. Many common expert systems have a reduced number of observable facts and the intelligence of the system is used only for the process of generating the prediction [6]. Most of them do not take into consideration the importance of this set of observable facts. The limited number of observable facts induces a generality problem that affects the quality of the observable facts.

Experts systems are capable of performing complex tasks in their specific field of expertise. They use a vast amount of explicit and tacit domain knowledge - domain ontology which should support problem solving method. A problem solving method is called an interface engine (in expert systems terminology).

The paper tries to show the idea of ontology semantic interpretation and related summary of used technologies, based on modern expert systems combined with ontological models platform. This concept combines main advantages of ontologies and expert systems - the ontology semantics and the rule based application used for searching information resources and issuing predictions similar like in [7].

\section{Expert System vs. Ontology}

The definition of ontology varies in the literature. A common understanding the ontology represents knowledge about the real word domain. The expert system is the system containing information about the problem domain in its knowledge base. Both of them are usable for knowledge management (defining, storing, searching, etc.). The common understanding of an expert system and ontology is very similar. Therefore, as the first step it is necessary to describe their definitions, their main characteristics and comparison.

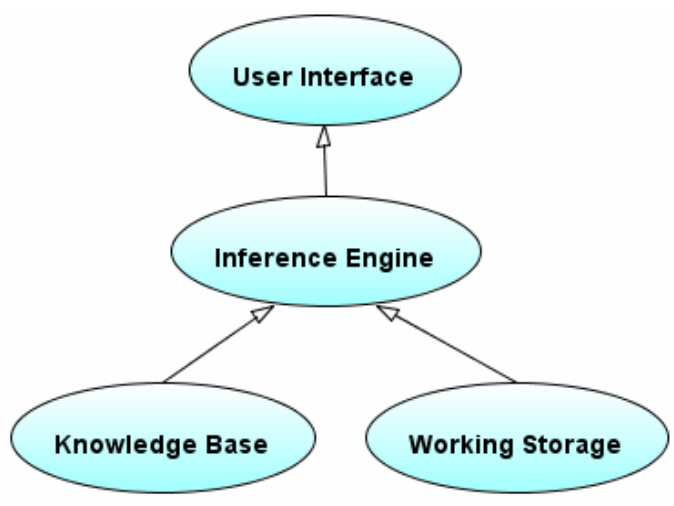

Fig. 2. Expert system schema

\subsection{Expert System}

Expert systems are mainly computer application which enables both algorithmic and non-algorithmic expertise for solving particular types of problems. Basically they are composed from four basic parts as is seen in Figure 2: 
- Knowledge Base - declarative representation of the expertise represented in IF ... THEN ... rules;

- Working Storage - the data that is specific to problem being solved;

- Inference Engine - the heart of the system which derives recommendation from the knowledge base and working storage;

- User Interface - component for input queries from users.

Expert systems recognize three basic types of knowledge:

- Descriptive (conceptual) knowledge (stored in knowledge base) - describes the concepts in the given domain and the relations among them. Each defined concept should be described by relation to already existing concepts.

- Procedural knowledge (stored in knowledge base) - clearly describes the procedures (actions) which should be processed in particular situation

- Factual knowledge (stored in working storage) - means the facts formalization. Especially facts describing particular situation or problem which should be solved

\subsection{Ontology}

Very precise definition of ontology in the context of computer and information science has been written by Tom Gruber [1]: "An ontology defines a set of representational primitives with which to model a domain of knowledge or discourse. The representational primitives are typically classes (or sets), attributes (or properties), and relationships (or relations among class members). The definitions of the representational primitives include information about their meaning and constraints on their logically consistent application." In the context of database systems, ontology can be viewed as a level of abstraction of data models, analogous to hierarchical and relational models, but intended for modeling knowledge about individuals, their attributes, and their relationships to other individuals.

\subsection{Expert System and Ontology Combination Advantages/Disadvantages}

It is necessary to accept the limitations of the technology upon which current knowledge-based systems are built. An inference engine alone doesn't provide an expert system. The main parts of each knowledge based systems are knowledge. As was mentioned in above text the limited number of observable facts induces a generality problem that affects the quality of the observable facts. Existence of many huge ontology models in various areas, their complexity and tolls supporting their development help to solve the lack of observable facts in expert systems based on ontology.

Moreover, it is usual in practice that two systems are using the same terms which mean different, sometimes completely contradictory, things. Ontology can help to pin down precisely what these differences are. More than serve as meta-data model, ontology can help to aggregation of more existing expert systems without loose of interoperability.

One of the tasks which have to be solved concerning SISE is to find the best way how to make ontology semantics available to the user using appropriate problem 
solving (search) interface. Experts systems are capable of performing complex tasks in their specific field of expertise, which should help to solve this task.

\section{Ontology Based Expert System}

As was stated in above text, the knowledge base should be the (SISE) ontology (mainly implemented in OWL). The main architecture concept of expert system based on ontology can be seen in the Figure 3 .

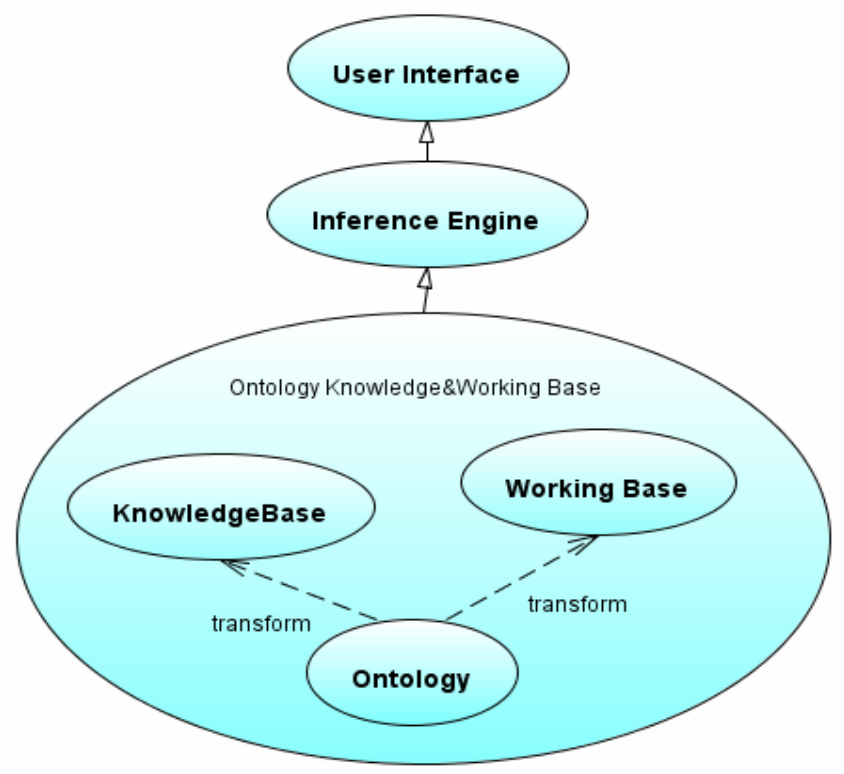

Fig. 3. Expert system based on ontology concept

The Ontology Knowledge\&Working Base is in fact the ontology model transformed to expert system knowledge and working base. The idea of transformation is described in the next part. The Inference Engine is the mechanism which applies the axiomatic knowledge present in the knowledge base to the problem-specific data leading to the conclusion. The User interface component is responsible for communication between user and expert system.

\section{Idea of Implementation}

For implementation idea will be used JESS the Rule Engine for the JavaTM Platform as main implementation language [8]; ROWL - Rule Language in OWL and Translation Engine for JESS [5] to transform ontology to expert system rules; and ontology written in OWL. 
As the first step is it is necessary to transform ontology (written in OWL in this case) to the knowledge and working base for expert system written in JESS. It can be done by using ROWL. It enables users to frame rules in RDF/XML syntax using an ontology in OWL. Using XSLT stylesheets, the rules in RDF/XML are transformed into forward-chaining defrules in JESS. The main principle of using ROWL can be seen on the Figure 4.

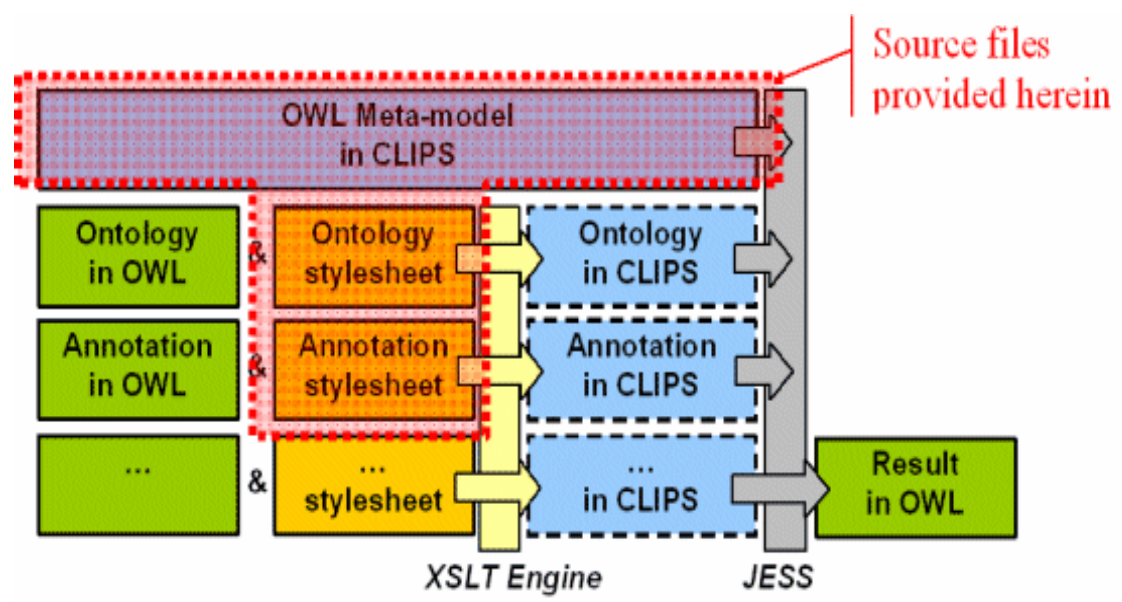

Fig. 4. OWL inference engine using XSLT and JESS - overall architecture [8]

Whole ontology model is transformed using style sheets (XSTL Engine) to ontology usable in JESS and can be used in expert system.

\section{Conclusion}

There was discussed the concept of combination of expert system and ontology in the paper. This concept is based on classical expert system architecture, where knowledge and working base is based on ontology. It has been discussed advantages and disadvantages of such combination and possibilities of this approach contribution to solve SISE task concerning finding the best way how to make ontology semantics available to the user. Finally, there were described the idea of implementation of this concept using new technologies (JESS, OWL, ROWL).

\section{References}

1. Gruber, T.: Encyclopedia of Database Systems, Liu, L., Tamer Özsu, M. (eds.). Springer, Heidelberg (2009)

2. Hřebíček, J., Kisza, K.: Conceptual Model of Single Information Space for Environment in Europe. In: Proceedings of the iEMSs Fourth Biennial Meeting: International Congress on Environmental Modelling and Software (iEMSs 2008), pp. 735-742. iEMSS, Barcelona (2008) 
3. Pillmann, W., Hřebíček, J.: Information Sources for a European Integrated Environmental Information Space. In: EnviroInfo 2009. Environmental Informatics and Industrial Environmental Protection: Concepts, Methods and Tools. 23. International Conference on Informatics for Environmental Protection, pp. 341-352. Shaker Verlag, Aachen (2009)

4. Hřebíček, J., Pillmann, W.: eEnvironment and the Single Information Space in Europe for the Environment. In: EnviroInfo 2010. 24th International Conference on Informatics in Environmental Protection, pp. 45-55. Shaker Verlag, Aachen (2010)

5. JESS - the Rule Engine for the JavaTM Platform, http: / /www . jessrules.com/

6. Merritt, D.: Building Expert Systems in prolog. Amzi! inc., Lebanon (2000)

7. Pariente, T., Fuentes, J.M., Angeles, M., González, S., Yurtsever, S., Avelino, G., Rizzoli, A., Nesic, S.: A Model for Semantic Annotation of Environmental Resources: The TaToo Semantic Framework. In: Hřebíček, J., Schimak, G., Denzer, R. (eds.) ISESS 2011. IFIP AICT, vol. 359, pp. 429-437. Springer, Heidelberg (2011)

8. ROWL: Rule Language in OWL and Translation Engine for JESS, http: / /mcom.cs.cmu. edu/OWL/ROWL/ROWL.html 